\title{
Laminar chaos in nonlinear electronic circuits with delay clock modulation
}

\author{
Thomas Jüngling, ${ }^{1, *}$ Thomas Stemler, ${ }^{1}$ and Michael Small ${ }^{1,2}$ \\ ${ }^{1}$ Complex Systems Group, Department of Mathematics and Statistics, \\ The University of Western Australia, 35 Stirling Highway, Crawley, WA 6009, Australia \\ ${ }^{2}$ Mineral Resources, CSIRO, Kensington, WA 6151, Australia
}

(Dated: January 10, 2020)

\begin{abstract}
We study laminar chaos in an electronic experiment. A two-diode nonlinear circuit with delayed feedback shows chaotic dynamics similar to the Mackey-Glass or Ikeda delay systems. Clock modulation of a single delay line leads to a conservative variable delay, which with a second delay line is augmented to dissipative delays, leading to laminar chaotic regimes. We discuss the properties of this particular delay modulation and demonstrate experimental aspects of laminar chaos in terms of power spectra and return maps.
\end{abstract}

\section{INTRODUCTION}

Delay systems have been studied in the last decades across various disciplines [1-4]. Mathematically, a delay system is a dynamical system with a retarded argument, which is significantly different from the corresponding undelayed system, for instance by its infinite dimensionality [5]. The close relationship between delay systems and spatially extended systems has been elaborated, and often the delay representation leads to new insights into the dynamical properties [4, 6-8]. A variety of physical origins leads to different types of delays, but a common characteristic is the signal propagation with finite speed between spatially separated parts of the system, or the temporary storage of information [9-11]. Nonlinear laser dynamics has been a major subject driving the field, because the fast timescales involved make the propagation delays inevitably relevant $[3,12]$. A second branch worth mentioning is time-delayed feedback control, in which a delay term acts as a desired control force [13-15].

From the plethora of delay models and corresponding real-world systems documented in the field nowadays, we distinguish the following classes. A simple (single-) delay system is given by $\dot{\mathbf{x}}(t)=\mathbf{f}(\mathbf{x}(t), \mathbf{x}(t-\tau))$, where $\mathbf{x} \in \mathbb{R}^{N}$ is the state vector of the system, which may be considered a network of low-dimensional oscillators. The function $\mathbf{f}$ is generally nonlinear, and often takes the form $\mathbf{f}(\mathbf{x}(t), \mathbf{x}(t-\tau))=\mathbf{f}_{0}(\mathbf{x}(t))+k \mathbf{f}_{\tau}(\mathbf{x}(t-\tau))$, where the delay term plays the role of feedback to a node, or coupling between different nodes in the network. One distinguishes between small, intermediate, or large delays, relative to a characteristic timescale of the dynamics, for instance a correlation or Lyapunov time, or the period of an orbit $[16,17]$. Extensions of the simple case are given by multiple delays, with either a finite or infinite number of delay terms, and variations in the arrangement of the different lags $\tau_{n}$ [18]. An example is the result of multiple reflections in a resonator, where $\tau_{n}=\tau_{0}+n \tau$, and the weights of the terms are exponentially decreasing with $n$. Further generalization leads to distributed delays, where

* thomas.jungling@uwa.edu.au the system typically contains a continuous delay kernel $g(\tau, \mathbf{x})$. The common property of the mentioned delay systems is that the delay dependency is constant.

A form of time-varying delays is given by state-dependent delays of the type $\dot{\mathbf{x}}(t)=$ $\mathbf{f}(\mathbf{x}(t), \mathbf{x}(t-\tau(\mathbf{x}(t))))$. This resembles a situation in which a node switches between inputs from other nodes at different distances [19, 20]. However, also the general form of delay modulation is being considered [21-24], and will be subject of our present investigation

$$
\dot{\mathbf{x}}(t)=\mathbf{f}(\mathbf{x}(t), \mathbf{x}(t-\tau(t))) .
$$

Typically, $\tau(t)>0$, and other constraints may apply depending on the situation, like continuity of the modulation, periodicity $\tau(t)=\tau(t+T)$, or a limited change rate $\dot{\tau}(t)<1$. In the limit of $T \rightarrow 0$ and a finite modulation amplitude, the distributed delay case is recovered, with the distribution reflecting the relative occurrence of delays from the modulation. The intermediate case of delay modulations on the timescale of the dynamics remains a challenging subject, in which novel phenomena can be expected due to the nontrivial interaction of the modulation, the mean delay, and the oscillatory modes of the nonlinear system. Recently, a distinction between conservative delays and dissipative delays has been introduced [25, 26]. A conservative delay modulation allows a transformation to a constant delay, whereas for dissipative delays this is not possible. Dissipative delays have been characterized by their signature in the Lyapunov spectrum.

Within dissipative delays, the same authors have subsequently discovered the phenomenon of laminar chaos [27]. Chaotic dynamics is common in delay systems and has been studied extensively for the case of large delays [28]. A distinction between strong and weak chaos has been made based on the sub-Lyapunov exponent of the delay system [29]. Weak chaos is a precondition for laminar chaos, because in strong chaos the leading instability is generated at the level of the nonlinear nodes driven by time-delayed signals, which makes them less susceptible to the effect of delay modulation. In essence, if the dissipation generated by the delay modulation overcomes the instability underlying the chaotic dynamics, 
the output of the system will show the laminar chaotic patterns. The dynamics in laminar chaos is characterized by subsequent plateaus of constant output, which vary in a chaotic way according to a nonlinear map, and switching between the plateaus is accompanied by a short irregular burst. Recently, also the first experimental evidence was reported in an electro-optic experiment with delay modulation from FPGA circuits [30].

In this work, we investigate laminar chaos in a fully electronic experiment with a focus on the locking regimes and the spectral properties. An electronic circuit with diode nonlinearities and digital delay lines allows for delay modulations which support both conservative and dissipative delays. The circuit generates chaotic dynamics in a wide parameter range, which for certain modulations of the delay leads to laminar chaos. We present and discuss the experimental characteristics of laminar chaos in terms of signatures in the power spectra, spectral entropy, and return maps. We further explore the relationship between delay modulation and chaotic instability by means of numerical simulations of the temporal dynamics, matching the experimental results with high accuracy. We finally discuss how to distinguish laminar chaos from similar experimental time series with a different mechanism. The theoretical basis is summarized in Sec. II as far as it applies to the experiment. The experimental setup is introduced in Sec. III, and the different experiments are described and discussed in Sec. IV, before we present our conclusions.

\section{THEORETICAL BACKGROUND}

We consider the variable-delay system according to Eq. (1) with a delay modulation of the form

$$
\tau(t)=\bar{\tau}+\hat{\tau} s(t) .
$$

The modulation is periodic with $s(t)=s(t+T)$, and the time dependence fulfills $-1 \leq s(t) \leq 1$ and $\langle s(t)\rangle_{t}=0$, where $\langle\cdot\rangle_{t}$ denotes the temporal average. The mean delay is $\bar{\tau}>0$, and with the modulation amplitude $\hat{\tau}<\bar{\tau}$ the delay is positive at all times. We adapt the notation in Ref. [27] and define the access map as the retarded argument of Eq. (1)

$$
R(t)=t-\tau(t)
$$

With the limitation $\dot{\tau}(t)<1$, the access map is invertible. The access map describes the times, at which the delayed signal originates, whereas the inverse access map $R^{-1}(t)$ refers to the future times at which a currently emitted signal will be injected into the dynamical system. Regardless of how the dynamical system maps its current input to the output, the inverse access map describes how a point in time iteratively visits the dynamical node. Assuming the signal transformation was explicitly given and summarized by a map $\mathbf{x}_{n}=\mathbf{F}\left(\mathbf{x}_{n-1}\right)$, where $\mathbf{x}_{n}=\mathbf{x}\left(t_{n}\right)$ and $\mathbf{x}_{n-1}=\mathbf{x}\left(t_{n}-\tau\left(t_{n}\right)\right)=\mathbf{x}\left(t_{n-1}\right)$, the corresponding positions in continuous time would be given by

$$
t_{n}=R^{-1}\left(t_{n-1}\right)
$$

The map $R^{-1}$ is a circle map, and as such exhibits either quasiperiodic dynamics or mode locking, which is quantified by a certain rotation number $\rho=\left\langle t_{n}-t_{n-1}\right\rangle_{n} / T$. These dynamical regimes define conservative and dissipative delays, respectively. Dissipation refers to the stability of the locking orbit, which means that there are absorbing points on the time axis which attract their neighborhood. Consequently, a stable orbit is accompanied by an unstable orbit, both originating from a tangent bifurcation, if the map is continuous. This means that in dissipative delays there is an interplay of expansion and contraction of time intervals both forward and backward in time. This property excludes a transformation of the time axis towards a constant-delay system, whereas for conservative delays such a transformation is possible, because despite finite-time distortions, all time intervals stay constant on average [25, 27].

When chaos occurs in a delay system, one distinguishes between strong and weak chaos [29]. This distinction is based on the sign of the leading sub-Lyapunov exponent (sub-LE), which is a measure of the conditional stability of the nonlinear node to the time-delayed feedback. Thus it does not directly depend on the type of delay, but is affected by the properties of the delayed signal, which in principle allows to switch between strong and weak chaos through the type of modulation. For the system considered in the present work, however, the sub$\mathrm{LE}$ is in good approximation a fixed property of the dynamical node and independent on the feedback signals. Moreover, the sub-LE is always negative, which corresponds to weak chaos and is a necessary condition for laminar chaos $[27,28]$. In weak chaos, the response of the dynamical node exhibits a functional dependency to its time-delayed drive, similar to the map $\mathbf{F}$ above, whereas strong chaos generates a level of inconsistency in the mapping between delay cycles [31-33]. It was shown that the emergence of laminar chaos depends on the competition between the temporal (horizontal) dynamics of the mode-locking access map, and the amplitude (vertical) dynamics of the chaotic return map. If the negative Lyapunov exponent (LE) of the access map overcomes the positive LE of the chaotic map, the dynamics collapses to a sequence of plateaus, where a short burst accompanies the switching between subsequent plateaus. This chaotic sequence of laminar phases has been defined as laminar chaos. If the access map is conservative, or if its dissipation is lower than the chaotic instability, no plateaus occur as if in ordinary delay chaos, and the dynamics has been coined turbulent chaos. 


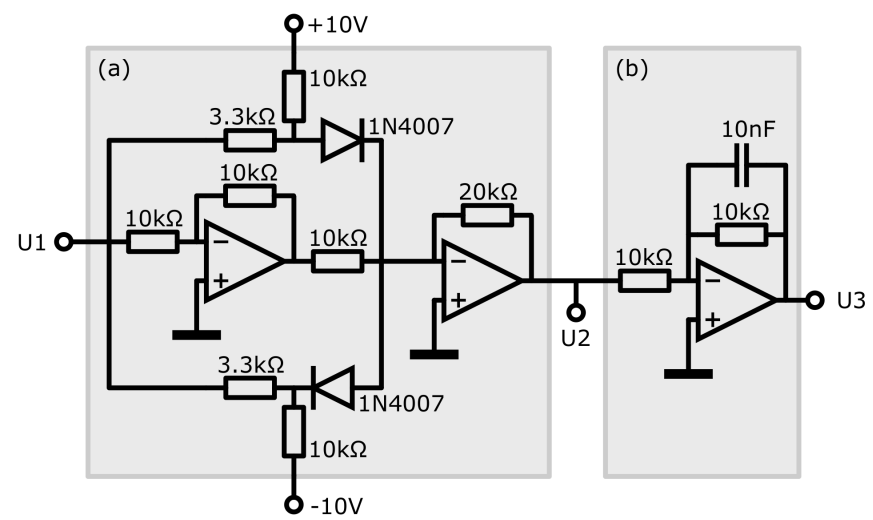

FIG. 1. Circuit diagram of the double-diode delay oscillator. Block (a): First processing stage with diode nonlinearities. Block (b): Second stage with low-pass filter. OP-amps are all TL084.

\section{EXPERIMENTAL SETUP}

Laminar chaos does not depend on the dimensionality of the system. Scalar delay systems have been a subject of investigation in the community throughout several decades. The Mackey-Glass system and the Ikeda system are well-studied candidates of this type [34-36]. We set up an electronic circuit experiment to realize a scalar delay system. The dynamical node of our circuit contains diodes as a source of nonlinearity. The circuit diagram of the nonlinear element is shown in Fig. 1. It consists of two signal processing stages. The first applies an instantaneous nonlinear function to the input, where instantaneous response means that the bandwidth of the circuit is much larger than the frequency range of typical signals. This bandwidth separation is guaranteed by the second stage, which is a dynamical element to introduce a characteristic timescale, for which we selected a low-pass filter with a sufficiently low cutoff frequency. Figure 2 (a),(b) shows the signal transformation after each stage. Two diodes connected in opposite direction create a symmetric nonlinearity out of their DC characteristics. The conduction threshold is shifted with a bias voltage, such that both diodes are not conducting in an input voltage range of about $-4 V<U_{1}<4 \mathrm{~V}$. Within this range, the circuit transmits linearly through an inverting OP amp with a total amplification factor of two, whereas outside the linear range the diodes effectively invert the slope of the map. This leads to a profile of two concatenated tent maps. The motivation for this design lies in the robust chaos found in the tent map, with little tendency to lock into a stable orbit. Moreover, slopes and extrema of the curve are set such that oscillations are constrained to the operating voltage ranges of all devices.

In order to generate a closed loop, we use delay lines as in Ref. [24]. The output $U_{3}$ of the dynamical element is connected to the input of the delay lines, and the output of the delay lines serves as the input voltage $U_{1}$
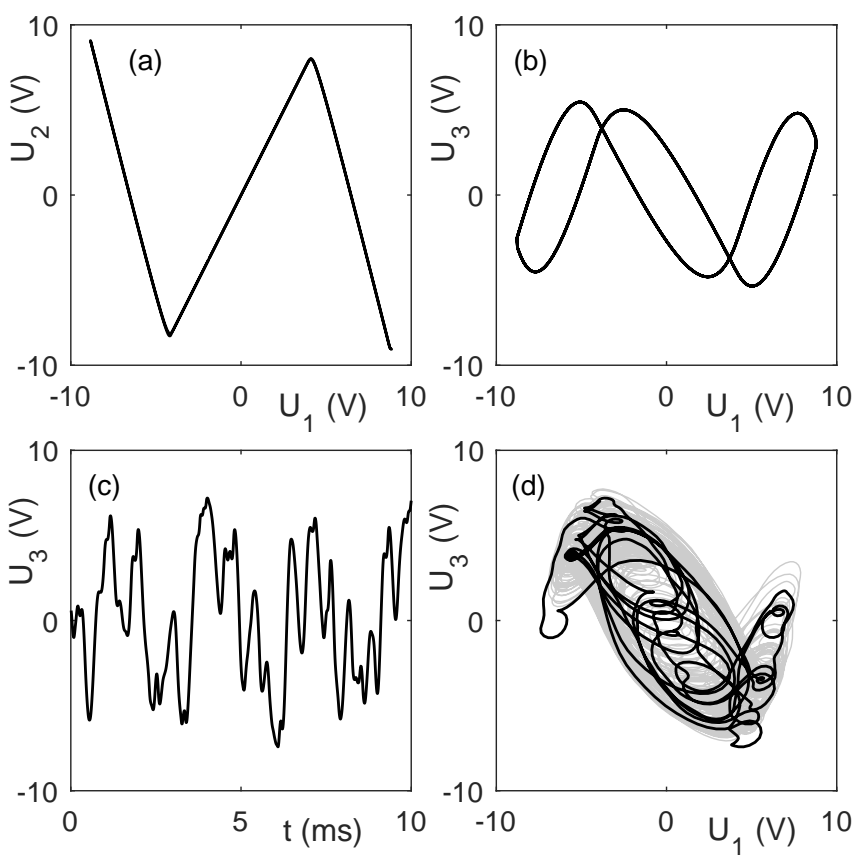

FIG. 2. Nonlinear and dynamical characteristics of the circuit in Fig. 1. Panels (a,b): response to a triangular waveform. Panels (c,d): chaotic delay dynamics. (a) Response of first stage, modulation $100 \mathrm{~Hz} 9 \mathrm{Vp}$. (b) Response of both stages with memory effect, modulation $500 \mathrm{~Hz} 9 \mathrm{Vp}$. (c) Output time series in chaotic regime with delay line $\tau=1.5 \mathrm{~ms}$. (d) Input vs. output in chaotic regime, time series same as in (c).

accordingly. The delay lines are digital, meaning that after analog conditioning an ADC converts the signal to 8bit resolution, a First In First Out (FIFO) memory with $1 \mathrm{kB}$ buffer size stores the information according to a write/read clock, and a DAC converts back to analog, which is then scaled and low-pass filtered. A tailored additive noise source exploits the dithering effect with the frequency band between the second-order filter at $20 \mathrm{kHz}$, and the clock frequency of up to $800 \mathrm{kHz}$, resulting in an effective resolution of maximal 11bit. A static clock frequency creates a constant delay $\tau=\tau_{0}+N / f_{c}$, where $N$ is the buffer size, $f_{c}$ is the clock frequency, and $\tau_{0} \approx 20 \mu s$ is an offset delay arising mainly from finite response times of all processing elements. Typical delay times are in the ms regime. The resulting equation of motion for the closed-loop system reads

$$
\dot{x}(t)=-\kappa x(t)+F(x(t-\tau)),
$$

where $x(t) \equiv U_{3}(t)$ is the scalar output voltage of the circuit, $\kappa$ is the time constant of the low-pass filter, and $F(\cdot)$ is the piecewise linear function as shown representing the dependency in Fig. 2 (a), which is directly applied to the delayed feedback. Due to the form of the instantaneous term, this system has always a negative sub-LE [37], thus any chaotic trajectory is of the weak chaos type, and the system is susceptible for laminar chaos. 
In most of the parameter settings of our experiment, the dynamics of the delay system settles on a chaotic attractor, such as is illustrated in Fig 2 (c),(d). Signals are recorded with a NI PXIe-6358 measurement device. We observe erratic waveforms with a limited bandwidth, where the limit originates mainly in the low-pass filter of the circuit with $\kappa / 2 \pi=1.6 \mathrm{kHz}$ cutoff. The delay here is set to $\tau=1.5 \mathrm{~ms}$, which is about double the filter time constant. Removing this filter, we would observe a similar dynamics with a higher frequency range, which is mainly limited by the bandwidth of the delay line. Plotting output of the circuit against its input yields an attractor representation which is familiar from similar chaotic delay systems. One can recognize the shape of the nonlinearity, blurred by oscillations from the dynamical elements.

Delay modulation can be achieved through modulation of the FIFO clock frequency, as the buffer size is fixed once at initialization. In principle, modulation of write clock and read clock separately would be possible, allowing for a wide range of modulation shapes within the restrictions of buffer size and maximum clock frequency. Our devices, however, allow only for a simultaneous modulation of write/read clock which are supplied from a common source. This constraint leads to a conservative delay modulation, regardless of buffer size and frequency modulation, which can be shown analytically as follows. By construction, the delay modulation for a given frequency modulation $f_{c}(t)$ and buffer size $N$ is given implicitly by

$$
N=\int_{R(t)}^{t} d t^{\prime} f_{c}\left(t^{\prime}\right) .
$$

Differentiation with respect to time yields

$$
\begin{aligned}
0 & =f_{c}(t)-f_{c}(R(t)) \dot{R}(t) \\
\Rightarrow \dot{R}(t) & =\frac{f_{c}(t)}{f_{c}(R(t))} .
\end{aligned}
$$

The slope of the access map determines the local rate of expansion or contraction of time intervals, and thus the stability of mode locking. We then follow a sequence of points in time according to Eq. (2). For an orbit with rotation number $p / q, q$ iterations of the access map coincide with $p$ modulation periods. Linearization along the orbit gives the product

$$
\begin{aligned}
\prod_{n=1}^{q} \dot{R}\left(t_{n}\right) & =\prod_{n=1}^{q} \frac{f_{c}\left(t_{n}\right)}{f_{c}\left(t_{n-1}\right)} \\
& =\frac{f_{c}\left(t_{q}\right)}{f_{c}\left(t_{0}\right)} \\
& =1 .
\end{aligned}
$$

The last step follows from $t_{q}-t_{0}=p T$, meaning that there is the same phase of the modulation after each orbit period. Thus every orbit of this map is marginally
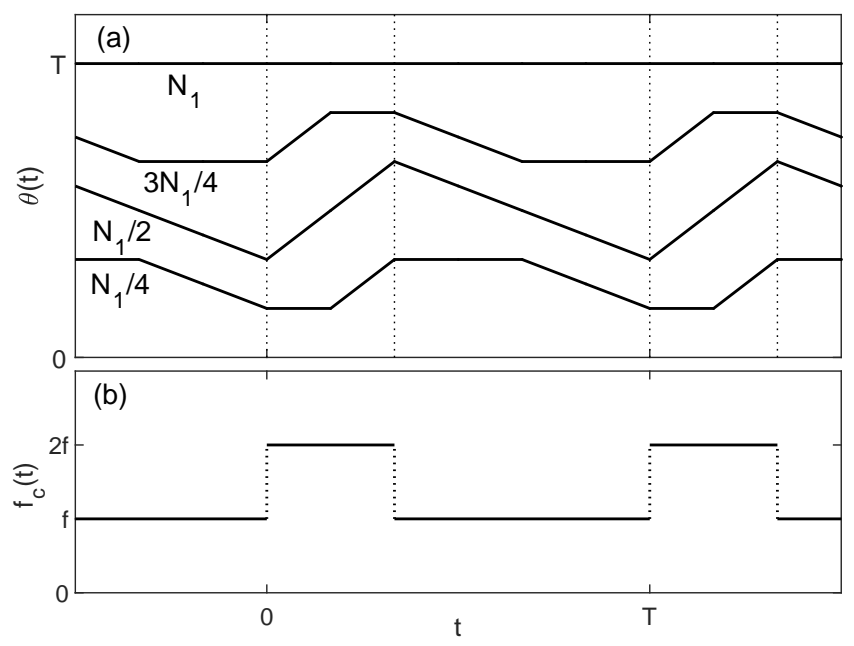

FIG. 3. Example of conservative delay modulation via clock frequency modulation $f_{c}(t)$ by a single delay line with buffer size $N$, according to Eq. (4). The modulation is periodic with period $T$. Panel (a): Resulting modulation of the forward delay time $\theta(t)=\tau\left(R^{-1}(t)\right)$ for buffer sizes $N=$ $N_{1} / 4, N_{1} / 2,3 N_{1} / 4, N_{1}$ (line labels), where $N_{1}$ is the integral over $f_{c}(t)$ for one period $T$ such that $N=N_{1}$ results in a constant delay $\theta(t) \equiv T \equiv \tau(t)$. Panel (b): Corresponding rectangular clock frequency modulation $f_{c}(t)$ with two levels, $f_{c} \equiv f$ for duration $T / 3$, and $f_{c} \equiv 2 f$ for duration $2 T / 3$. Switchings are indicated in panel (a) as vertical dotted lines.

stable. Alternatively, one may consider an arbitrary orbit. Even if initial and final frequency do not coincide, they are bound by their maximum ratio. Since this is the maximum multiplicator for an arbitrary number of iterations, the average growth rate must be zero. Here, we assume that $f_{c}$ is strictly positive. We discuss briefly the case of zero clock frequency at the end of Sec. IV.

We illustrate the conservative delay time modulation of a single delay line by the example shown in Fig. 3. The clock frequency is modulated in a rectangular way, switching between a base frequency, $f_{c}(t) \equiv f$, and double the frequency. The higher frequency remains active for one third of the modulation cycle $T$, and the lower frequency for the other two third. We measure the resulting delay modulation by the forward delay $\theta(t)$, which is the transit time that a signal recorded at time $t$ will spend until it passes the delay line at $R^{-1}(t)$. This transit time is related to the access map by $\theta(t)=R^{-1}(t)-t$, and with the delay via $\theta(t)=\tau\left(R^{-1}(t)\right)$. The limitation $\dot{\tau}<1$ in this representation reads $\dot{\theta}(t)>-1$. The delay depends on the buffer size $N$ through Eq. (4). When the buffer equals the integral over one period $T$, which we denote as $N=N_{1}$, the delay becomes constant $\theta(t) \equiv \tau(t) \equiv T$. The delay modulations for $N=N_{1} / 4, N_{1} / 2,3 N_{1} / 4$ are $T$-periodic triangular waveforms with clipping, where the maximum amplitude is achieved for the middle value. For $N>N_{1}$, the patterns repeat in the same way. The only intersection of $\theta(t)$ with multiples of $T$, which cor- 
responds to periodic orbits of the access map, is when $\theta$ is a constant, which is equivalent to the delay being conservative. This includes also higher orbits, because iterations of the access map correspond to integer multiples of $N$. Physically, this is related to the fact that the output of the delay line is connected to its input via the nonlinear circuit, and independently of the nonlinear dynamical properties the sampling rate at both points is identical. This situation is equivalent to adding further identical delay lines to the loop while maintaining the conservative delay modulation.

\section{EXPERIMENTS}

Chaotic dynamics with a conservative delay modulation is not significantly different from the constant-delay dynamics as shown in Fig. 2. The modulation signatures appear in the power spectrum, and in the time series one can observe stretched and squeezed intervals depending on the depth of the delay modulation, see Fig. 4 (a). These patterns are finite-time phenomena which are unrelated to genuine laminar chaos. A simple method to arrive at dissipative delays using our devices is by adding a second delay line serially, which creates a constant offset delay $\tau_{0}$. Such a delay is already inherently present in each delay line, and in principle it would be sufficient to push the access map through a shifted bisectrix such that mode locking occurs. For delay offsets close to zero, however, the locking becomes structurally unstable, so that an artificial increase of $\tau_{0}$ is desired. In this configuration, and with a sinusoidal frequency modulation of the variable delay line, we were able to achieve laminar chaos. The typical waveforms are shown in Fig. 4 (b), (c).

In order to get an overview over the locking regimes, we investigate a range of different offset delays $\tau_{0}$. The mean clock frequency of the variable delay line is chosen as $\bar{f}_{c}=200 \mathrm{kHz}$, and a sinusoidal frequency modulation $f_{c}(t)=\bar{f}_{c}+\hat{f}_{c} \sin (2 \pi t / T)$ is applied with an amplitude of $\hat{f}_{c}=190 \mathrm{kHz}$ and a period of $T=10 \mathrm{~ms}$. This leads to a delay modulation around $\bar{\tau} \approx 5 \mathrm{~ms}$ with an amplitude of $\hat{\tau} \approx 2 \mathrm{~ms}$. Note that this delay modulation contains harmonics which would form the conservative shape if only the single delay line was used. By iteration of the corresponding access map for the total delay of both lines, we first determine numerically the locking regimes. Figure 5 shows the resulting Lyapunov multiplier $\mu_{R}$ of the map as a function of the buffer size $N$ of the modulated delay line, and the offset $\tau_{0}$ from the additional constant delay. The multiplier is the exponential of the corresponding LE, $\mu_{R}=\exp \left(\lambda_{R}\right)$, and as such the geometric mean of the slopes of the inverse access map evaluated along the sequence of points $t_{n}$ in time from Eq. (2),

$$
\lambda_{R}=\left\langle\log \left|\dot{R}^{-1}\left(t_{n}\right)\right|\right\rangle_{n} .
$$

The choice of the multiplier over the exponent is more convenient for our setting. A multiplier of $\mu_{R}=1$ cor-
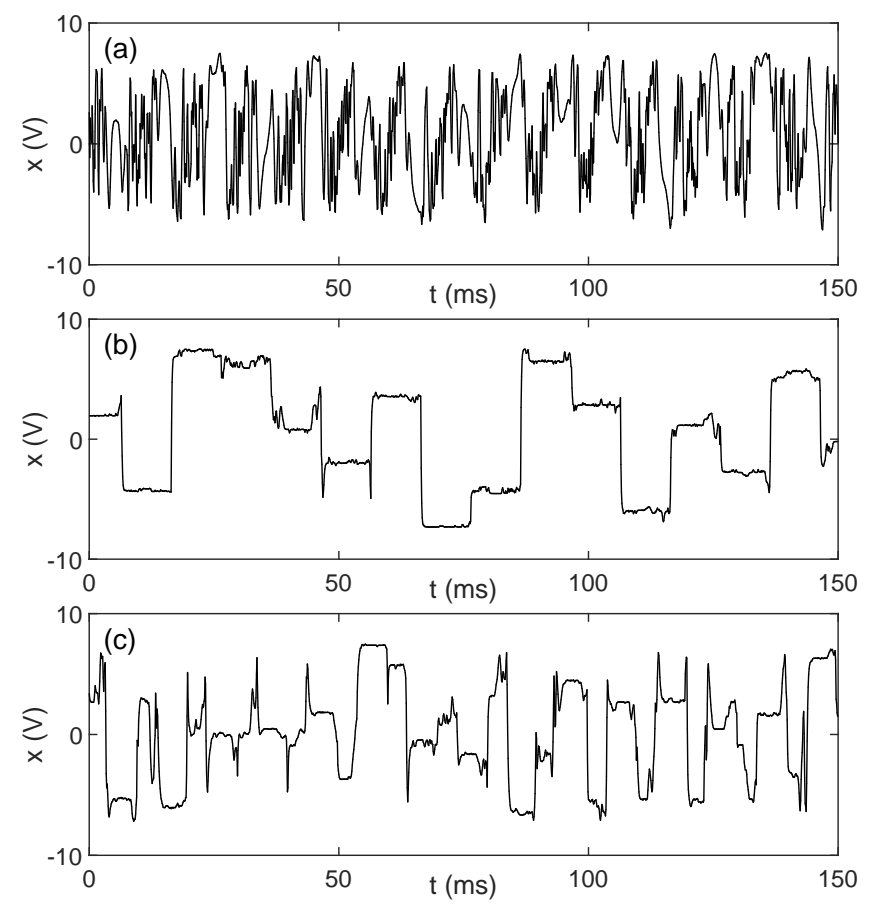

FIG. 4. Sample trajectories in turbulent (a) and laminar chaos (b,c) for selected parameters as shown in Fig. 5. Modulation period $T=10 \mathrm{~ms}$. Rotation numbers are $\rho=1$ (b) and $\rho=1 / 2(\mathrm{c})$. Signals $x(t)$ are the voltages $U_{3}(t)$ of the circuit.

responds to conservative delays, whereas $0 \leq \mu_{R}<1$ are dissipative delays with mode locking on a rational rotation number. The laminar chaos condition expressed in multipliers reads $\mu_{R} \mu_{F}<1$, meaning that the contraction on the time axis due to stable orbits of $R^{-1}$ overcomes the expansion from the chaotic map $F(\cdot)$ from Eq. (3) which acts on the system trajectory. The displayed range shows the first 'Brillouin zone', which is given by $0 \leq N \leq N_{1}$ and $0 \leq \tau_{0} \leq T$. The value of $N_{1}=2000$ corresponds to the aforementioned case in which the buffer covers exactly one modulation period and thus leads to a constant delay $\tau(t) \equiv T$. On the $\tau_{0^{-}}$ axis, a delay offset equal to the period $T$ maintains the type of modulation. Thus the boundaries of the domain are conservative delays, and the same pattern repeats for multiples of $N_{1}$ and $T$, in $N$ and $\tau_{0}$, respectively. The center of the domain shows a large locking regime with rotation number $\rho=1$. The second largest structures are tongues with $\rho=1 / 2$ (lower left) and $\rho=3 / 2$ (upper right), followed by smaller higher-order tongues. Remarkably, there is a pronounced tendency to suppress all mode locking in the vicinity of the domain boundaries. We interpret this behavior as a general feature of circle maps, which are constructed as a combination of a conservative map and an offset.

For selected values of the buffer size $N$, we conduct the electronic experiment and record time series for a range of $\tau_{0}$. By means of the power spectra, we summarize the 


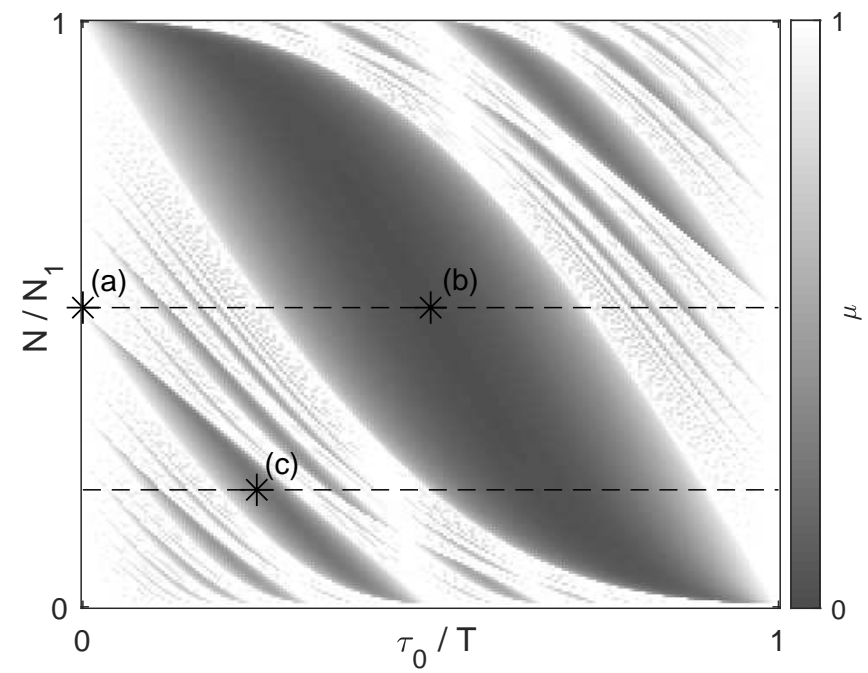

FIG. 5. Numerically determined Lyapunov multiplier $\mu_{R}$ (grayscale) as a function of the variable delay line buffer size $N$ and the delay offset $\tau_{0}$ from the second delay line. Standard sinusoidal clock frequency modulation is applied, see text. Axes are normalized to the values $N_{1}$ and $T$, corresponding to the full modulation period. Markers (a)-(c) indicate parameter settings for time series samples in Fig. 4. Upper and lower horizontal dashed line correspond to experimental section in Fig. 6 and Fig. 7, respectively.

dynamics for $N=1023$ in Fig. 6 . The frequency range shown includes the first few harmonics of the modulation. There are clear bands around $\tau_{0}=5 \mathrm{~ms}$ and $\tau_{0}=15 \mathrm{~ms}$, in which there are spectral dropouts at integer multiples of the modulation frequency $100 \mathrm{~Hz}$. These signatures correspond to laminar chaos of the dominant mode locking with $\rho=1$, as the wavelengths of the plateau size are suppressed. The situation in the next locking windows is the same, as they appear at multiples of the modulation period. Differences in the remaining spectral bands indicate the higher rotation numbers, which effectively correspond to multiple phases of the return map being iterated in parallel. In the turbulent chaos bands between the locking regimes, the situation seems opposite. There are peaks related to the mean delay time, which manifests in their hyperbolic traces. The fact that the first peak shows at half the delay frequency, $1 /\left(2\left(\tau_{0}+\bar{\tau}\right)\right)$, is related to the negative input-output correlation which appears at one loop cycle. In the laminar chaos regimes, these peaks disappear in favor of the dip signatures, whereas most of the spectral power accumulates in a broad band at low frequencies, corresponding to the timescale of the chaotic dynamics between the plateaus. Finally, the timescale of the low-pass filter shows in the slow overall decline of the spectrum in this range, which is less than half of the cutoff frequency.

The corresponding section of the numerically determined Lyapunov multiplier supports the experimental findings. In order to estimate the boundaries of laminar

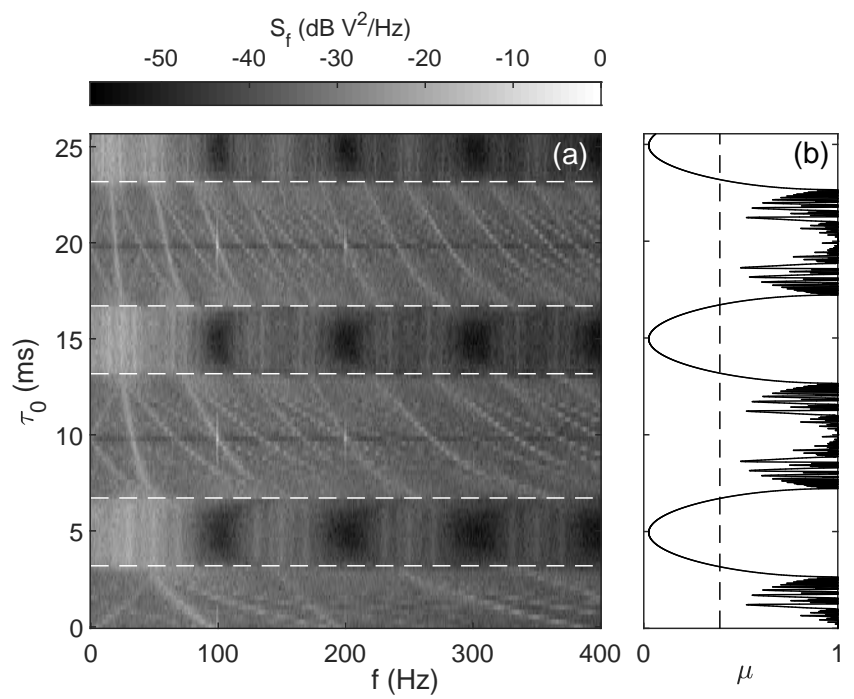

FIG. 6. Turbulent and laminar chaos, depending on delay offset $\tau_{0}$, see text. Panel (a): Power spectra with intensity (grayscale) normalized to the highest peak in the shown range. Frequency resolution is $1 \mathrm{~Hz}$. Panel (b): Corresponding Lyapunov multiplier $\mu_{R}$ of the access map for the modulation settings determined numerically. Vertical dashed line: Inverse of the estimated average slope $\mu_{F}^{-1}$ of return map as shown in Fig. 2 (a). Horizontal dashed lines in panel (a) correspond to intersections of multipliers in (b).

chaos, we determine the Lyapunov multiplier of the return map $\mu_{F}$. The piecewise slopes in Fig. 2(a) are determined, and a weighted average assuming a uniform distribution in the typical amplitude range reveals $\mu_{F}=2.55$. Laminar chaos as given by $\mu_{R}<\mu_{F}^{-1}$ is indicated by the constant threshold. The determined regions are in excellent agreement with the experimental results.

We repeat the experiment for a section at $N=400$, as indicated in Fig. 5. Based on the same calculations as before, we expect to observe the robust locking at integer values of $\rho$, and the side bands with $\rho=p / 2$ at odd $p$. The power spectra are again calculated as before and summarized by the spectral entropy $H_{S}$, see Fig. 7 . We indeed observe clear drops in the entropy at the corresponding locations, corresponding to the lower bandwidth due to lower number of active degrees of freedom in laminar chaos. The integer locking regimes are more pronounced because of the lower value of $\mu_{R}$, which leads to a suppression of noise in the plateaus of the time series. See snapshots of the time domain in Fig. 4. Noise is inherently present in the experiment and originates from various sources. A detailed investigation of laminar chaos and noise is given in Ref. [30].

The presence of noise, together with the time series as the only access to the dynamics of the system, motivates the question about the reliability of the identification of laminar chaos, and the accuracy of the location of the bifurcation points. Moreover, our setup allows for phases with zero clock frequency, in which even for a single de- 

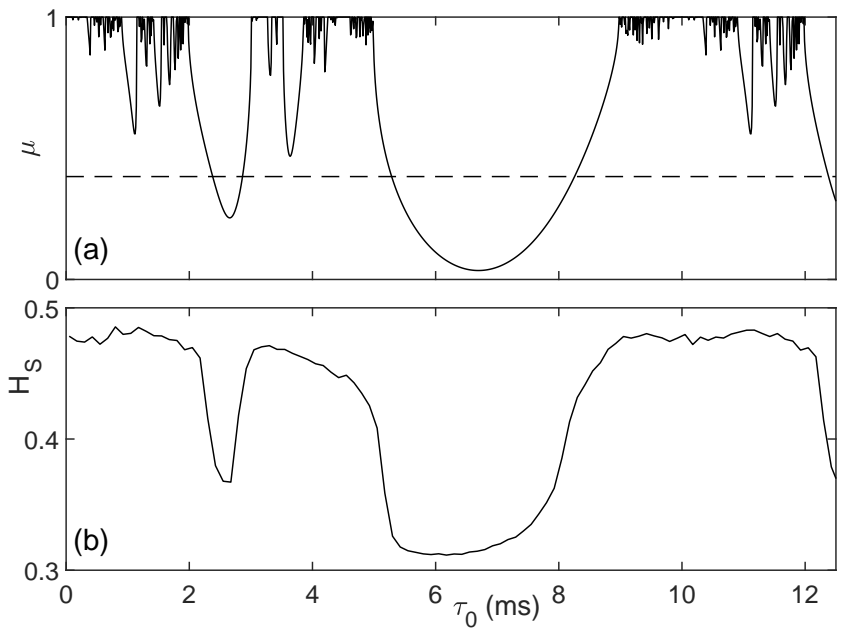

FIG. 7. Panel (a): Numerical Lyapunov multiplier $\mu_{R}$ (solid line) and $\mu_{F}^{-1}$ (dashed line). Panel (b): Spectral entropy $H_{S}$ from experiments. Standard modulation (sinusoidal, see text) with $T=10 \mathrm{~ms}$ corresponds to section $N=400\left(N_{1}=2000\right)$ in Fig. 5.

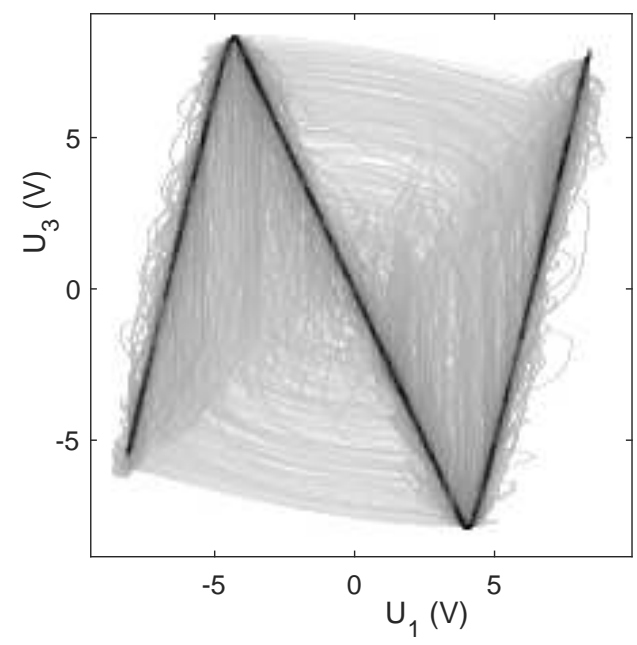

FIG. 8. Input-output representation of laminar chaotic trajectory. The grayscale encodes the relative frequency of the dynamics visiting a point of the return portrait, with black (white) corresponding to the maximal (minimal) frequency.

lay line a constant output can be artificially generated, despite the delays being conservative. Depending on the settings, such waveforms might look similar to the laminar chaos outputs. A means to identify laminar chaos in experiment, and to distinguish it against pseudo-laminar chaos from paused delay lines, is the input-output map as shown in Fig. 8. The figure shows a grayscale plot of the trajectory, in which the intensity represents the relative frequency spent at a point. One can recognize the profile of the static map of Fig. 2 clearly contrasted against a background of transients. These correspond to the plateaus and the bursts of laminar chaos, respectively. Each plateau corresponds to a single point on the static map, accumulating to the entire portrait. The return map is largely robust against noise, because most of the fluctuations on the plateau are slow as compared to the bursts, thus contributing to the emergence of the static map. In contrast, for the pseudo-laminar chaos case the corresponding portrait would be entirely blurred (not shown). This is the result of several iterations of the nonlinear map occurring in the remaining clipping regimes of the access map, see Fig. 3, such that the simple return map is never found between any subsequent modulation cycles, whereas laminar chaos always reveals a return map between corresponding iterates of the access map.

\section{CONCLUSION}

We have implemented an electronic circuit experiment demonstrating laminar chaos. A double diode nonlinearity with a low-pass filter forms the nonlinear dynamical element. Digital delay lines with a bandwidth in the hearable range allow for chaotic dynamics with delay modulation. We have shown that using a single such delay line leads to only conservative delays because of the simultaneous write and read clock modulation. An additional delay line creates a delay offset which allows to access the dissipative delays. The special composition of the delay modulation leads to characteristic suppression regimes of dissipation in the vicinity of the main conservative mapping. We have found laminar chaotic patterns within the most robust locking regimes in excellent agreement with numerical results using only properties of the access map and the chaotic return map. Moreover, we have analyzed spectral properties of laminar and turbulent chaos, and shown that the spectral entropy is a useful indicator of both dynamics. Finally, we have discussed how the laminar chaotic return map is distinguished from other similar dynamical patterns in conservative delay lines with paused outputs.

\section{ACKNOWLEDGEMENTS}

M.S. is supported by Australian Research Council Discovery Project (Grant No. DP180100718).
[1] W. Just, A. Pelster, M. Schanz, and E. Schöll, Phil. Trans. R. Soc. A 368, 303 (2010).
[2] M. Wolfrum, S. Yanchuk, P. Hövel, and E. Schöll, Eur. Phys. J. Spec. Top. 191, 91 (2010). 
[3] M. C. Soriano, J. García-Ojalvo, C. R. Mirasso, and I. Fischer, Rev. Mod. Phys. 85, 421 (2013).

[4] S. Yanchuk and G. Giacomelli, J. Phys. A: Math. Theor. 50, 103001 (2017).

[5] K. Ikeda and K. Matsumoto, Physica D 29, 223 (1987).

[6] G. Giacomelli, R. Meucci, A. Politi, and F. T. Arecchi, Phys. Rev. Lett. 73, 1099 (1994).

[7] G. Giacomelli and A. Politi, Phys. Rev. Lett. 76, 2686 (1996).

[8] F. Marino and G. Giacomelli, Phys. Rev. Lett. 122, 174102 (2019).

[9] S. Campbell, "Time delays in neural systems," in Handbook of Brain Connectivity, Understanding Complex Systems, edited by V. Jirsa and A. McIntosh (Springer, Berlin, Heidelberg, 2007) pp. $65-90$.

[10] G. Orosz, R. E. Wilson, R. Szalai, and G. Stépán, Phys. Rev. E 80, 046205 (2009).

[11] P. Fleurquin, J. J. Ramasco, and V. M. Eguiluz, Sci. Rep. 3, 1159 (2013).

[12] A. Argyris, D. Syvridis, L. Larger, V. Annovazzi-Lodi, P. Colet, I. Fischer, J. Garcia-Ojalvo, C. R. Mirasso, L. Pesquera, and K. A. Shore, Nature 438, 343 (2005).

[13] K. Pyragas, Phys. Lett. A 170, 421 (1992).

[14] K. Pyragas, Phys. Lett. A 206, 323 (1995).

[15] W. Just, T. Bernard, M. Ostheimer, E. Reibold, and H. Benner, Phys. Rev. Lett. 78, 203 (1997).

[16] S. Yanchuk and P. Perlikowski, Phys. Rev. E 79, 046221 (2009).

[17] M. Lichtner, M. Wolfrum, and S. Yanchuk, SIAM J. Math. Anal. 43, 788 (2011).

[18] A. Ahlborn and U. Parlitz, Phys. Rev. E 72, 016206 (2005).

[19] J. Mallet-Paret and R. Nussbaum, Arch. Rational Mech. Anal. 120, 99 (1992).

[20] J. Martínez-Llinàs, X. Porte, M. C. Soriano, P. Colet, and I. Fischer, Nature Comm. 6, 7425 (2015).
[21] S. Madruga, S. Boccaletti, and M. A. Matías, Int. J. Bifurc. Chaos 11, 2875 (2001).

[22] A. Gjurchinovski and V. Urumov, Europhys. Lett. 84, 40013 (2008).

[23] A. Gjurchinovski and V. Urumov, Phys. Rev. E 81, 016209 (2010).

[24] T. Jüngling, A. Gjurchinovski, and V. Urumov, Phys. Rev. E 86, 046213 (2012).

[25] A. Otto, D. Müller, and G. Radons, Phys. Rev. Lett. 118, 044104 (2017).

[26] D. Müller, A. Otto, and G. Radons, Phys. Rev. E 95, 062214 (2017).

[27] D. Müller, A. Otto, and G. Radons, Phys. Rev. Lett. 120, 084102 (2018).

[28] H. Wernecke, B. Sándor, and C. Gros, Phys. Rep. 824, 1 (2019).

[29] S. Heiligenthal, T. Dahms, S. Yanchuk, T. Jüngling, V. Flunkert, I. Kanter, E. Schöll, and W. Kinzel, Phys. Rev. Lett. 107, 234102 (2011).

[30] J. D. Hart, R. Roy, D. Müller-Bender, A. Otto, and G. Radons, Phys. Rev. Lett. 123, 154101 (2019).

[31] A. Uchida, R. McAllister, and R. Roy, Phys. Rev. Lett. 93, 244102 (2004).

[32] N. Oliver, T. Jüngling, and I. Fischer, Phys. Rev. Lett. 114, 123902 (2015).

[33] T. Jüngling, M. C. Soriano, N. Oliver, X. Porte, and I. Fischer, Phys. Rev. E 97, 042202 (2018).

[34] M. Mackey and L. Glass, Science 197, 287 (1977).

[35] K. Ikeda, Optics Comm. 30, 257 (1979).

[36] K. Ikeda, H. Daido, and O. Akimoto, Phys. Rev. Lett. 45, 709 (1980).

[37] T. Jüngling, M. C. Soriano, and I. Fischer, Phys. Rev. E 91, 062908 (2015). 